\title{
An ounce of prevention
}

\author{
Stephen B Hanauer
}

I've just returned from organizing and chairing a 4-day symposium for primary care physicians by specialists from the University of Chicago's Department of Medicine. The meeting was enlightening from beginning to end: starting with updates on cardiopulmonary medicine and ending with discussions about polypharmacy in the elderly. Most prominently, I was impressed by the fact that the majority of chronic and/or lethal diseases in our society are preventable.

Starting with heart disease, which is the number one killer of US men and women, the vicious cycle of obesity/diabetes/metabolic syndrome/sleep apnea/hypertension prevails across most specialties. Add cigarette smoking and excess alcohol consumption to the mix and we can include oropharyngeal and lung cancers, along with cancer of the esophagus, pancreas, and perhaps breast. Alcohol is the primary cause of chronic liver disease and an accelerant to becoming infected with HBV (sexually transmitted) and HCV (intravenous drug abuse).

One can certainly appreciate the problems with the US health system (as exemplified by Michael Moore's movie "Sicko"), expected to become a major point of debate in the next Presidential election, but right now our society needs to focus on preventing illness. In my mind, disease prevention and global warming are two main issues of our generation (in this regard, some might say that even the war in Iraq can be traced to our reliance on oil, which so greatly contributes to

\section{... right now our society needs to focus on preventing illness.}

SB Hanauer is Editorin-Chief of Nature Clinical Practice Gastroenterology \& Hepatology.

Competing interests
The author declared no
competing interests.
Reprinted from
Hanauer SB (2007) Nat Clin
Pract Gastroenterol Hepatol
4: 583
www.nature.com/clinicalpractice
doi:10.1038/ncpgasthep0960

environmental issues). Unfortunately, current health-care debates focus on complex and difficult political, economic and administrative issues. By contrast, disease prevention can be very simple.

I was struck by the statistic that the average size of fast food hamburgers or portion size of french fries has doubled in the past 30 years. In addition, highly calorific food additives (sugar, fructose, corn starch, hydrogenated fats) have become commonplace in processed foods. That the US Government subsidizes tobacco, corn and sugar while eliminating support for physical education in schools is self-defeating!

We can easily reduce the strain on our health-care system by concentrating on improving our health rather than paying at the 'back end' to treat preventable diseases. In contrast to developing countries where famine and infectious diseases are the primary killers of infants and children, in our society an overabundance of food and abuse of alcohol, drugs and tobacco are leading to chronic illnesses that are sopping up health-care resources. We need to focus on childhood obesity and stop subsidizing unhealthy products. Let's teach our children to eat healthily, add exercise back into our daily routines, and tax cigarettes to levels that equate to the added health-care costs that their consumption causes. From heart disease to polypharmacy in the elderly, it's pathetic that we are paying at the back end for the treatment of preventable diseases. 